\title{
DESCRIPTIVE STUDY OF BABIES ABOVE 36 WEEKS GESTATION DISCHARGED ON HOME OXYGEN - WHAT DO WE KNOW?
}

\author{
Kristina Chmelova, Hae Sung Kim, Sridhar M Ramaiah, Naveen Athiraman
}

Neonatal Unit at Royal Victoria Infirmary, Newcastle upon Tyne, UK

\section{Background}

Term infants represent a minority of all babies discharged home on oxygen therapy from our Neonatal Intensive Care Unit (NICU). This group is important to be recognised as impact of home oxygen therapy might be changing their long term outcomes and quality of life. However, data is insufficient. We aimed to study characteristics of near term infants who were discharged on home oxygen (gestational age 36- 42 weeks).

\section{Results}

There were a total of 2171 near term admissions to NICU during the 7 year study period (2010-2016). Among them, $31(1.4 \%)$ were discharged on home oxygen. Among them, 16/31 (52\%) required respiratory support soon after the birth. Most of them were born by caesarian section 19/31 (62\%). We identified meconium aspiration syndrome 8/31 (26\%), HIE 3/31 (9.6\%) and respiratory distress syndrome $3 / 31(9.6 \%)$ as most common diagnoses. Others included PPHN, pneumothorax, syndromes and congenital anomalies. The median hospital stay was 14 days (range 10-31 days, IQR 10.5). Median oxygen need in the community was 13 weeks (range 2-37 weeks, IQR 8).

\section{Methods}

In this restrospective audit project, infants discharged home on oxygen from NICU were identified from all near term admissions (over 36 weeks of gestation). Data on background diagnosis, length of hospital stay, duration of home oxygen and immediate outcomes were collected.

\section{Respiratory support required prior discharge}

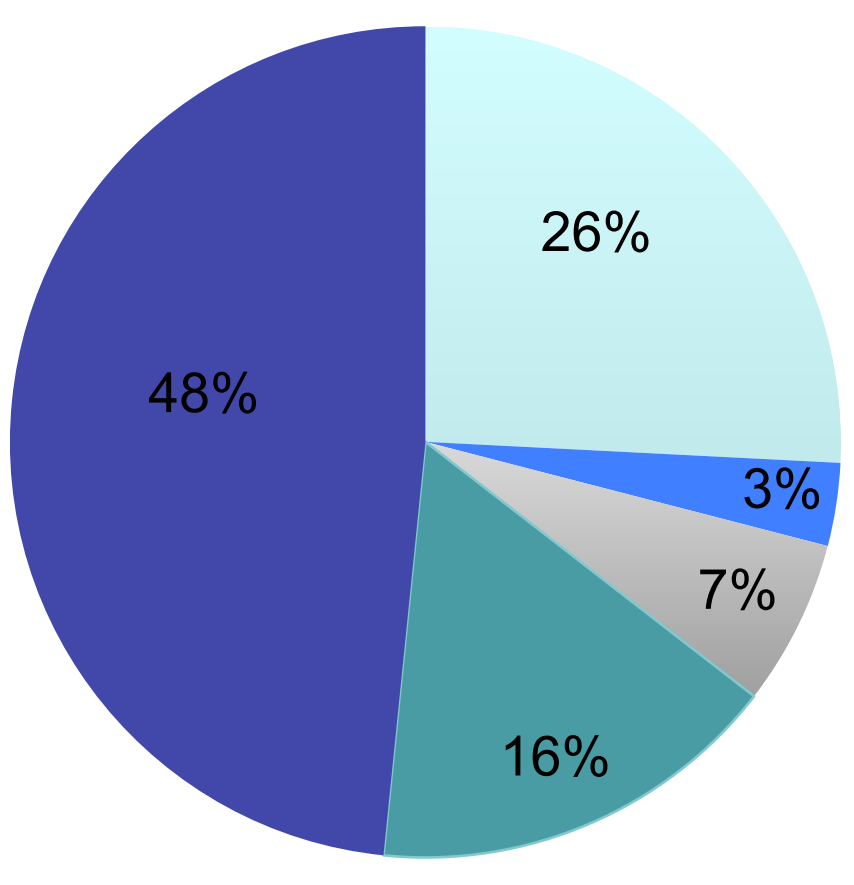

Ventilation + LF O2

$\square \mathrm{HF}+\mathrm{CPAP}+\mathrm{LF}$ $\mathrm{O} 2$

Ventilation + CPAP + LF O2

- CPAP + LF O2

- LF O2

\begin{tabular}{rr}
\hline Conditions & Percentage \\
\hline Meconium aspiration syndrome & $26 \%$ \\
Hypoxic ischaemic encephalopathy & $9,6 \%$ \\
Congenital cardiac anomalies & $9,6 \%$ \\
\hline Persistent pulmonary hypertension of the newborn & $6,4 \%$ \\
Prader Willi syndrome & $6,4 \%$ \\
Trisomy 21 & $6,4 \%$ \\
Pneumothorax & $6,4 \%$ \\
\hline Venous sinus thrombosis & $6,4 \%$ \\
\hline
\end{tabular}

\section{Conclusion}

A small proportion of infants born $>36$ weeks require oxygen at home. Around two thirds of these infants were born by caesarian section and most common diagnosis was meconium aspiration syndrome. The oxygen weaning process 